Classification

Physics Abstracts

42.80

\title{
Système de traitement acousto-optique de signaux numériques
}

\author{
P. Goutin, P. Logette, J. M. Rouvaen et E. Bridoux \\ Laboratoire d'Opto-Acousto-Electronique, URA 832 C.N.R.S., Université de Valenciennes, \\ 59326 Valenciennes Cedex, France
}

(Reçu le 8 mars 1991, accepté le 4 juillet 1991)

\begin{abstract}
Résumé. - L'élément fondamental du traitement numérique du signal est le filtre transversal. Parmi les techniques utilisables, la convolution acousto-optique est particulièrement bien adaptée à cette fónction. Les difficultés de cette opération, pour le traitement d'un signal d'entrée relativement long, proviennent de la configuration propre du convoluteur et du phénomène de propagation. Celles-ci vont nécessiter l'étude d'un algorithme de calcul respectant certaines contraintes. La réalisation du processeur hybride nous a permis d'en évaluer les caractéristiques à partir des résultats expérimentaux tels que : autoconvolutions, filtrage F.I.R., corrélations, codes de Golay, codes de Barker et multiplication matricielle. Le fait même de la nature prototype du système réalisé nous amène à présenter un grand nombre d'extensions et d'améliorations qui permettent d'optimiser fortement la vitesse de traitement.
\end{abstract}

\begin{abstract}
The transversal filter is a fundamental device in numerical signal processing. Among all available techniques, the acousto-optic convolution is very well matched to this function. Some penalties occur however, for very long input signals, owing to the physical configuration of the convolver and to the propagation of acoustic waves in its bulk. This calls for the study of a convenient computation algorithm taking all the constraints into account. The realization of an hybrid processor enabled us to evaluate its main characteristics using experiments like autoconvolution, F.I.R. filtering, Golay's and Barker's codes or matrix multiplication. Since the built system is actually a prototype, a number of extensions and improvements are presented, which may lead to the optimization of the processing speed.
\end{abstract}

\section{Introduction.}

Le but du traitement de signal est de faire ressortir au mieux ce que l'on désire observer (le signal) d'un environnement parasite (le bruit). Cette fonction de base est le filtrage pris dans son sens le plus large. Actuellement, la plupart des systèmes de mesures fournissent des informations sous forme de signaux électriques et le niveau technologique de l'électronique en matière d'échantillonnage et de numérisation permet de transformer ces dernières en une suite de valeurs numériques. A condition de prendre certaines précautions pour effectuer convenablement cette opération, ce type de traitement présente les avantages d'une reproductibilité totale, d'une précision conservée tout au long du calcul, d'une stabilité à long terme (pas de vieillissement) et à court terme (pas de dérive) ainsi que d'une grande souplesse 
d'utilisation par simple modification d'un programme. En outre, il permet de réaliser des fonctions ne possédant pas d'équivalent analogique.

\section{Présentation du filtre transversal.}

L'élément fondamental du traitement numérique est le filtre transversal. Sa structure est donnée dans la figure 1 [1]. les échantillons $e(i)$ du signal transitent dans une ligne à retard assurant la fonction de mémorisation d'un nombre $n$ fini d'échantillons. Ce nombre $n$ définit la longueur du filtre. Les prises intermédiaires permettent de disposer des répliques du signal à des instants successifs dont la sommation pondérée par les coefficients $c(j)$ réalise la fonction de convolution discrète suivante :

$$
s(i)=\sum_{j=0}^{n} c(j) \cdot e(i-j) .
$$

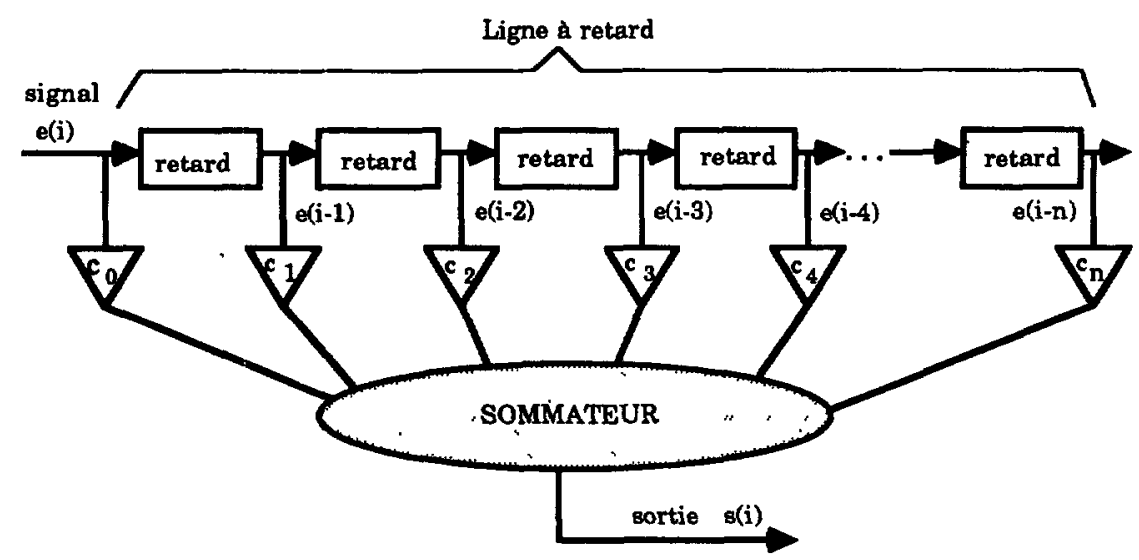

Fig. 1. - Filtre transversal.

[Transversal filter.]

De nombreuses technologies ont été utilisées pour réaliser de tels systèmes. Les composants à transfert de charges ont une structure bien adaptée au filtrage transversal. Le plus connu, le CCD (Charge Coupled Device), présente l'inconvénient d'une faible bande passante [2] et son utilisation principale est dans le domaine de la vidéo.

Dans la gamme des circuits intégrés, une grande variété de composants de traitement de signal (CTS), développés par de nombreux constructeurs (Texas-Instrument, Nec, Motorola, Intel, Analog Devices, .. et en Europe Thomson et Philips) est apparue sur le marché. Parmi ceux-ci, on trouve des CTS programmables, dont la configuration peut être facilement adaptée et des CTS spécialisés (dédiés) qui favorisent une application particulière. Dans les deux cas, le principe de base est le même et peut se résumer par la figure 2 dans lequel on retrouve la ligne à retard (registre à décalage) et la pondération/sommation (multiplieuraccumulateur).

Les techniques mettant en œuvre les ondes acoustiques sont de plus en plus à l'ordre du jour. L'interaction acousto-électronique non linéaire entre ondes acoustiques de surface, intéressante pour sa bande passante, est trop figée dans sa structure liée à la géométrie des transducteurs $[3,4]$. Le même problème se rencontre pour l'interaction acousto-optique en 


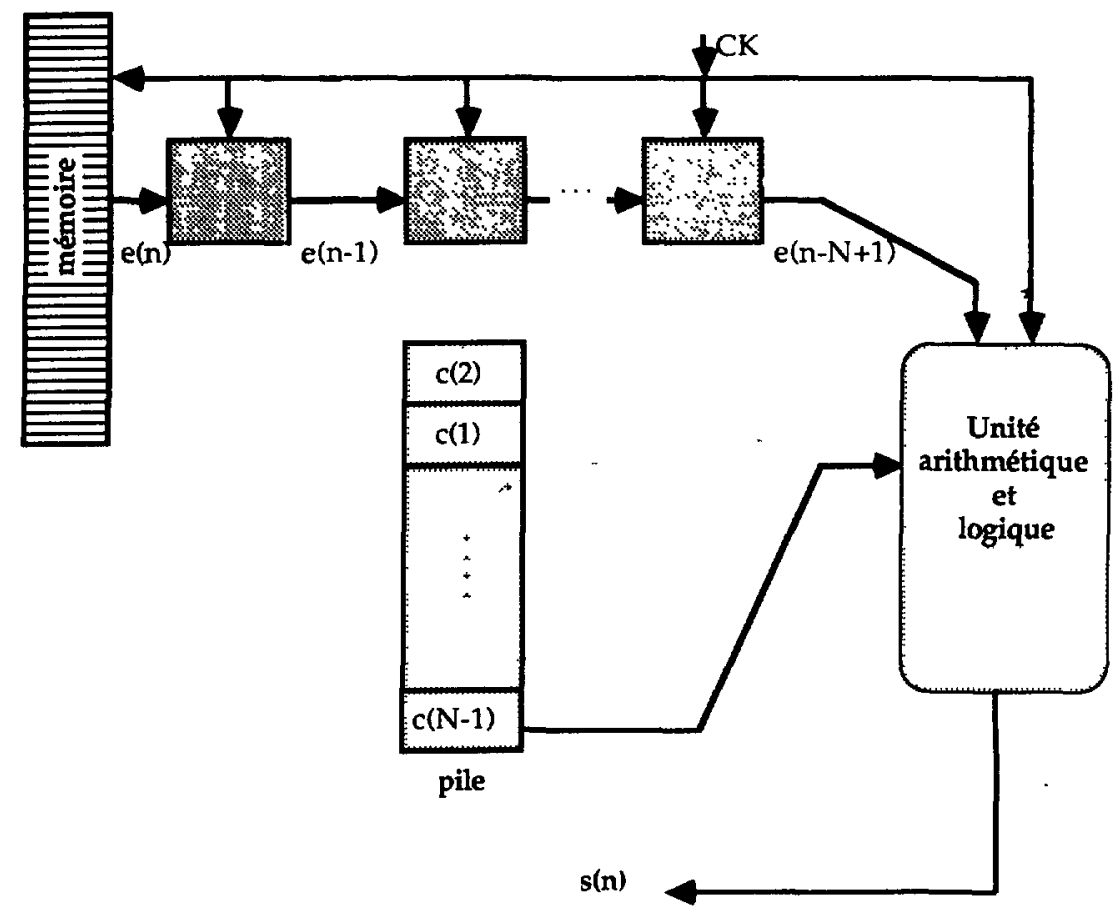

Fig. 2. - Principe d'un CTS.

[DSP principle.]

ondes de surface qui présente un grand rendement dû à la densité d'énergie élevée de l'onde acoustique. Cette difficulté peut être cependant contournée par l'emploi d'ondes de volume.

La nécessité d'une grande souplesse d'utilisation liée au fonctionnement du filtre transversal justifie le choix de cette dernière méthode. Dans la partie suivante, nous ferons des rappels sur l'interaction en régime de Bragg et la configuration en convoluteur. Nous présenterons, ensuite, le système [5] qui a été réalisé au laboratoire ainsi que quelques résultats obtenus. Nous terminerons par les solutions susceptibles d'en améliorer les performances.

\section{La fonction acousto-optique.}

L'interaction entre une onde acoustique et une onde lumineuse a été étudiée pour la première fois par Brillouin en 1922 et les premières expériences ont été effectuées dès 1932 par Lucas et Biquard, en France. La mise en œuvre de nouvelles technologies a permis le développement d'applications de l'acousto-optique en offrant la possibilité d'une grande vitesse de modulation et de déviation d'un faisceau lumineux.

Le principe général de l'interaction acousto-optique est le suivant : une onde acoustique engendre, dans le matériau où elle se propage, une suite de compressions et de dilatations qui vont induire localement des variations de l'indice optique. Nous pouvons distinguer deux cas principaux selon la largeur du faisceau ultrasonore et la valeur du rapport des longueurs d'ondes acoustique $\left(\lambda_{\text {op }}\right)$ et lumineuse $\left(\Lambda_{\mathrm{ac}}\right)$ [6] : le cas limite de Raman-Nath, dans lequel plusieurs ordres diffractés peuvent coexister et celui de Bragg où nous n'obtiendrons qu'un seul faisceau diffracté. Sous cette dernière condition, le faisceau diffracté est dévié d'un angle $\pm \lambda_{\mathrm{op}} / \Lambda_{\mathrm{ac}}$ et sa pulsation vaudra $\omega_{\mathrm{op}} \pm \Omega_{\mathrm{ac}}$. En inclinant d'un angle $\theta_{\mathrm{B}}$, appelé angle de Bragg, 


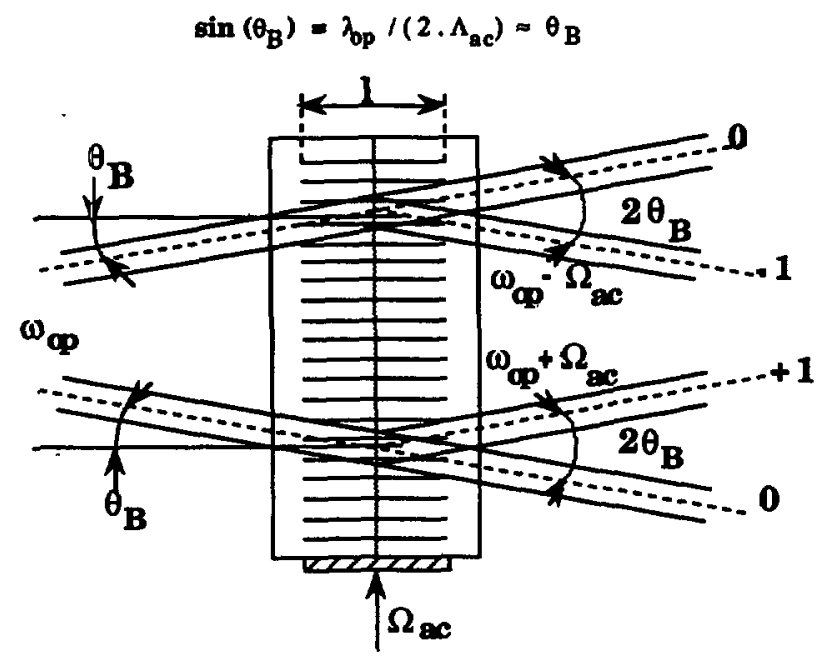

Fig. 3. - Interaction acousto-optique en régime de Bragg.

[Acousto-optic Bragg interaction.]

le faisceau lumineux perpendiculairement au vecteur d'onde acoustique (Fig. 3) nous favorisons l'un des deux angles précédents (ordre \pm 1 ). Cet angle doit respecter la condition $[3,7]$ :

$$
\sin \left(\theta_{\mathrm{B}}\right)=\lambda_{\mathrm{op}} / 2 \cdot \Lambda_{\mathrm{ac}} .
$$

Si la puissance acoustique appliquée, $P_{\text {act }}$, est faible, l'intensité du faisceau dévié lui est proportionnelle. On peut donc moduler l'intensité de celui-ci en faisant varier la puissance appliquée au transducteur. C'est donc la structure de base utilisée pour réaliser un modulateur acousto-optique.

Considérons un premier modulateur dans lequel on envoie un signal ultrasonore de pulsation $\Omega_{\text {ac }}$ et d'amplitude $A_{1}(t)$, dans les conditions de Bragg, en favorisant l'ordre +1 par exemple. Positionnons un deuxième modulateur parallèle au premier mais dans lequel une seconde onde acoustique se propage en sens opposé à la première. La pulsation de cette onde acoustique est également $\Omega_{\mathrm{ac}}$, mais son amplitude est $A_{2}(t)$. Suite à la seconde interaction, nous disposons d'un faisceau non défléchi que nous appellerons $\mathbf{E}^{00}$, de deux faisceaux défléchis une seule fois $\left(\mathbb{E}^{0-1}\right.$ et $\mathbf{E}^{10}$ ) et du faisceau $\mathbf{E}^{11}$ doublement défléchi, mais dont les déviations se compensent, qui ressort parallèle au faisceau $\mathbf{E}^{00}$ (Fig. 4) et dont la pulsation sera $\omega_{\text {op }}+2 . \Omega_{\text {ac }}[8]$.

$\mathrm{Au}$ travers d'une lentille sphérique, les ordres $\mathbf{E}^{00}$ et $\mathbf{E}^{11}$ se superposent au niveau du plan focal, où nous disposerons un photodétecteur. La réponse quadratique de ce dernier donnera un signal de sortie de la forme:

$$
i(x, t)=\left(\mathbf{E}^{00}+\mathbf{E}^{11}\right)^{2}
$$

avec $\quad \mathbf{E}^{00}=a \cdot A_{0} \cdot \sin \left(\omega_{\mathrm{op}} t\right)$

et $\quad \mathbf{E}^{11}=b \cdot A_{0} \cdot A_{1}(t, x) \cdot A_{2}(t, x) \cdot \sin \left[\left(\omega_{\mathrm{op}}+2 \cdot \Omega_{\mathrm{ac}}\right) t+\phi\right]$

et où la dépendance en $x$ représente la distribution spatiale du faisceau ( $a, b$ étant des facteurs de proportionnalité dépendant en particulier de l'opacité des milieux). Compte tenu des 


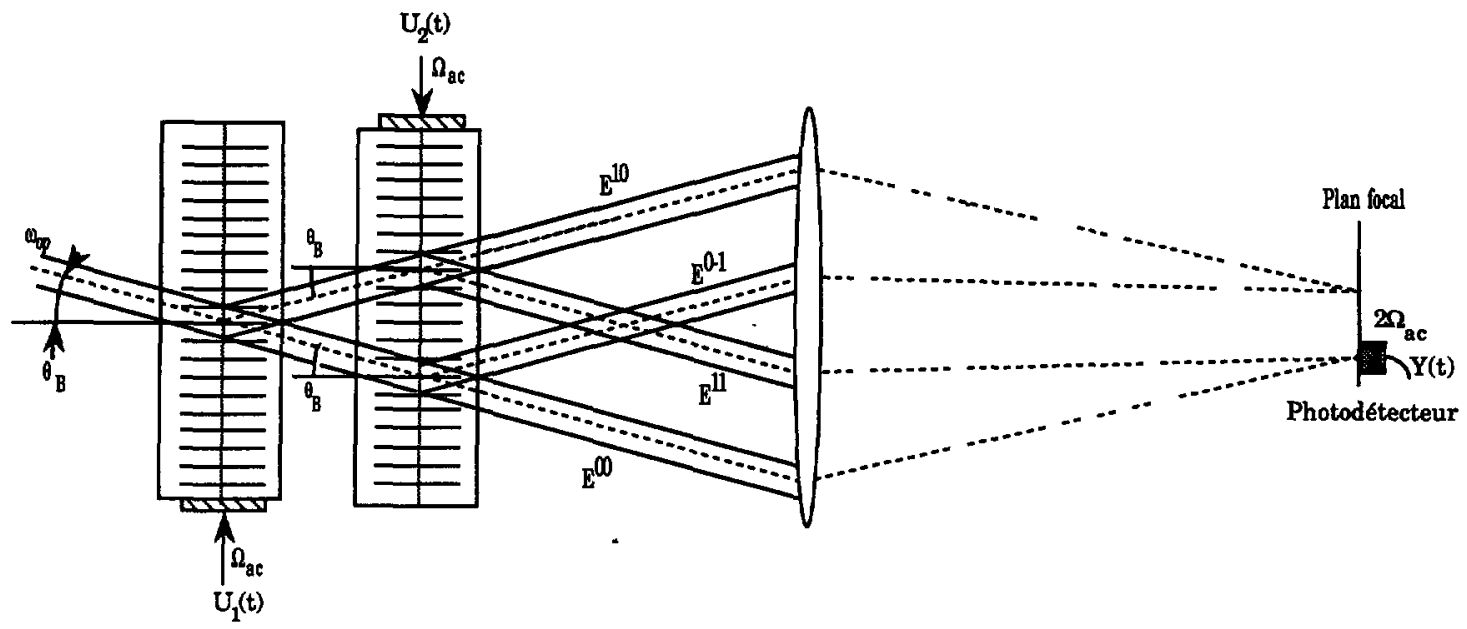

Fig. 4. - Convoluteur acousto-optique.

[Acousto-optic convolver.]

fréquences présentes et du temps de réponse du détecteur, l'expression globale du signal de sortie peut se mettre, après changement de variable, sous la forme:

$$
i(t)=\cos \left(2 \cdot \Omega_{\mathrm{ac}} t+\phi\right) \cdot \int A_{1}(2 \cdot t-y) \cdot A_{2}(y) \cdot \mathrm{d} y
$$

qui apparaît alors comme un signal de pulsation porteuse $2 . \Omega_{\text {ac }}$, modulé par la convolution des signaux acoustiques affectée d'une compression d'un facteur 2 dans le temps.

Si nous remplaçons les signaux de modulation continus par des signaux échantillonnés nous aboutissons pour le signal détecté, à une relation de convolution discrète qui décrit, comme nous l'avons déjà vu (1), le fonctionnement d'un filtre transversal.

\section{Principe de l'algorithme de base.}

Nous présentons ici l'algorithme originel employé lors de la réalisation du système déjà existant au laboratoire [9]. Les difficultés principales sont liées à la configuration en convoluteur du système acousto-optique (sens de propagation inverses) et au phénomène de propagation lui-même.

EFFET DU FACTEUR D'ÉCHELlE. - Nous avons souligné la contraction dans le temps d'un facteur 2(4) lors de la convolution acousto-optique. Au niveau de la transformation discrète, ce phénomène se concrétise par le fait que, au cours d'une même propagation, les points calculés sont de même parité. Ceci suppose que l'injection des échantillons et la lecture des résultats se font à la même fréquence. En effet, en observant la figure 5 qui décrit le déroulement des séquences durant deux coups d'horloge successifs, nous constatons que, si au coup d'horloge $p$, nous disposons en sortie de :

$$
s(q+6)=e(q+6) \cdot c(0)+e(q+5) \cdot c(1)+e(q+4) \cdot c(2)+e(q+3) \cdot c(3)+\cdot
$$
au coup d'horloge $p+1$, nous obtiendrons :

$$
s(q+8)=e(q+8) \cdot c(0)+e(q+6) \cdot c(1)+e(q+5) \cdot c(2)+e(q+4) \cdot c(3)+\cdot
$$

Suivant la parité de $q$, nous obtiendrons tous les résultats pairs ou impairs. Pour le calcul des termes de l'autre parité, il suffira de décaler une des émissions d'un coup d'horloge par rapport à l'autre émission (Fig. 6). Le traitement s'effectuera donc en deux temps désignés par passes. 

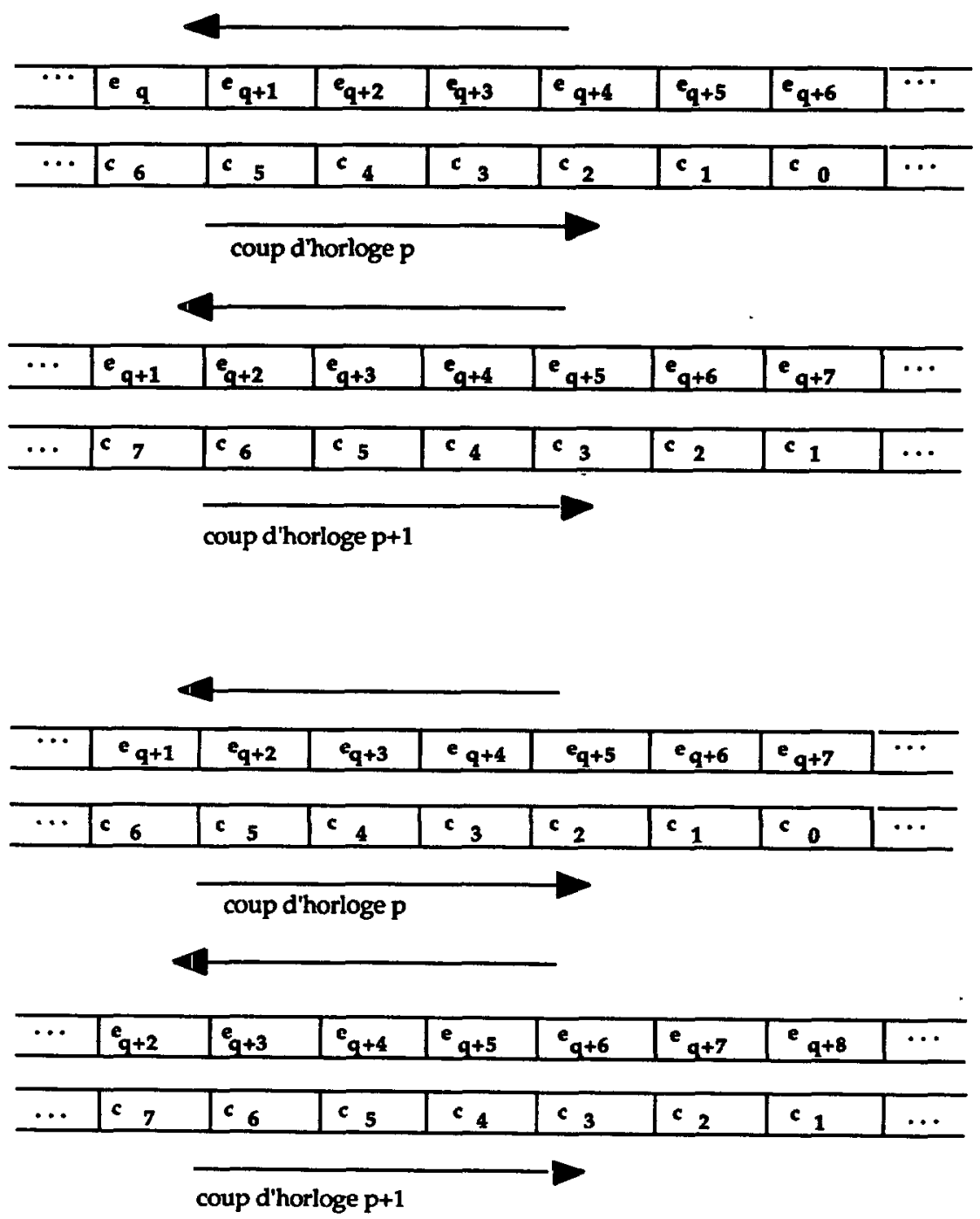

Figs. 5 et 6. - Effet du-facteur d'échelle.

[Effect of the timing scale.]

EFFET DO A LA PROPAGATION. - Le phénomène de propagation, pour une largeur $L$ finie d'interaction, limite les nombres $N_{\mathrm{e}}$ d'échantillons du signal d'entrée et $N_{\mathrm{c}}$ de coefficients du filtre transversal désiré, simultanément présents dans les modulateurs. Cette valeur maximale $N_{\text {cel }}$, appelée nombre de cellules du modulateur, est définie par:

$$
N_{\text {cel }}=L \cdot f_{\mathrm{e}} / v
$$

où $f_{\mathrm{e}}$ est la fréquence d'injection des échantillons et $v$ la vitesse de propagation de l'onde acoustique.

Ce nombre correspond à la longueur maximale $\left(\dot{N}_{\mathrm{c}}\right.$ maximal) du filtre FIR réalisable par le convoluteur acousto-optique dans son principe de base. Pour effectuer un calcul complet, chacune des deux passes précédentes nécessite une décomposition en plusieurs passages, ou 
sous-passes. Néanmoins, au cours d'une même sous-passe, plusieurs échantillons résultats successifs seront disponibles.

REPONSE TEMPORELLE DISCRÈTE DU FIR. — Le filtre FIR de réponse impulsionnelle $\{c(j)\}$ et de longueur $N_{\mathrm{c}}$, soumis à une suite d'échantillons d'entrée $\{e(k)\}$, restitue donc à sa sortie, un signal $\{s(i)\}$ décrit par:

$$
s(i)=\sum_{j=0}^{N_{\mathrm{c}}-1} c(j) \cdot e(i-j) .
$$

Les cas où $(i-j)$ est inférieur à 0 appartiennent au régime transitoire et, plus précisément, correspondent au temps de montée du filtre. Ceux où (i) est supérieur au nombre $N_{\mathrm{e}}$ d'échantillons du signal d'entrée correspondent au temps de descente du filtre. Autrement, nous sommes en régime permanent. La taille du signal de sortie est de $N_{\mathrm{e}}+N_{\mathrm{c}}-1$.

\section{Description de l'algorithme.}

Afin d'optimiser la vitesse de traitement, il faut profiter pleinement du nombre $N_{\text {cel }}$ de cellules des modulateurs. Pour cela, au cours d'une sous-passe, les émissions des échantillons doivent être synchronisées de telle sorte que, en régime permanent, le premier instant de présence simultanée des différents coefficients corresponde à la sortie du premier résultat de cette sous-passe.

Il faut distinguer le régime transitoire qui est calculé lors de la première et de la dernière sous-passes de chaque passe, et le régime permanent calculé dans les autres sous-passes. Ceci va se répercuter sur le séquencement de l'algorithme puisque les longueurs des sous-passes considérées ne sont pas identiques.

Après diverses simulations menées en vue de généraliser l'algorithme, nous constatons qu'il est avantageux de commencer le cycle de calcul par une passe paire si $N_{\mathrm{c}}$ est impair et par une passe impaire autrement. Ainsi, nous ne parlons plus que de première et deuxième passes.

Nous détaillons ici les étapes temporelles importantes de l'algorithme qui sont la durée des sous-passes, l'instant de libération des coefficients et la prise en compte des résultats.

DURÉE DES SOUS-PASSES. - Cette durée se décompose en deux temps. Le premier est attribué au parcours du premier échantillon signal émis sur toute la longueur d'interaction; il vaut donc $N_{\text {cel }}$ coups d'horloge. A L'issue de ce dernier, le premier résultat du régime permanent est disponible. Le second correspond au temps durant lequel ceux-ci restent simultanément présents, soit $N_{\text {cel }}-N_{\mathrm{c}}$. Nous avons donc une durée totale de $2 N_{\text {cel }}-N_{\mathrm{c}}$. Dans le cas spécifique de la première sous-passe de la seconde passe, il faut incrémenter cette durée d'un coup d'horloge afin de tenir compte du décalage introduit à l'émission des échantillons pour les résultats de l'autre parité (Fig. 6).

LibERATION DES COEFFICIENTS. - Pour définir cet instant relativement à l'émission du premier échantillon signal, nous raisonnons sur la base du résultat précédent. En effet, la fin des sous-passes coïncide avec l'arrivée du premier coefficient en fin de longueur d'interaction. Il aura donc été émis $N_{\text {cel }}$ coups d'horloge auparavant. Ceci détermine donc les instants de libération des coefficients; dans le cas particulier de la première sous-passe de la seconde passe, nous avons $N_{\text {cel }}-N_{\mathrm{c}}+1$ et dans les autres cas, $N_{\text {cel }}-N_{\mathrm{c}}$.

PRise EN COMPTE DES RÉSUltats. - Dans ce cas, l'instant de référence est l'instant de libération des coefficients. En régime permanent, le premier résultat est disponible dès la présence simultanée de tous les coefficients décrivant le filtre. Comme nous l'avons vu dans la 
partie précédente, la prise en compte intervient au bout de $N_{\mathrm{c}}$ coups d'horloge. Reste à considérer maintenant les régimes transitoires de début et de fin d'une durée de $N_{\mathrm{c}}-1$ chacun. Le temps de descente qui se produit à la fin du calcul, n'intervient qu'au niveau du nombre total d'échantillons résultat à prendre en compte $\left(N_{\mathrm{e}}+N_{\mathfrak{c}}-1\right)$. En ce qui concerne le temps de montée, il faut distinguer les deux parités des résultats dont le calcul s'effectue au cours de la première sous-passe de chacune des passes. Si $N_{\mathrm{c}}$ est impair, la répartition du temps de calcul se fait de façon égale pour les deux sous-passes $\left(N_{\mathrm{c}}-1\right.$ est pair). Autrement, après analyse du problème, on peut montrer que la première sous-passe de la première passe donne $N_{\mathrm{c}} / 2$ résultats transitoires et que celle de la seconde restitue les $N_{\mathrm{c}} / 2-1$ manquants [10].

\section{Réalisation pratique du système.}

Le système peut être scindé en deux éléments principaux : la partie analogique/optique, correspondant à la mise en œuvre du convoluteur, et la partie purement numérique qui concerne le pilote et sa configuration.

Le montage optique et anAlogique. - La partie optique comprend le laser de type monomode suivi d'un jeu de lentilles pour obtenir un pinceau lumineux, puis les éléments actifs du montage qui sont les deux modulateurs acousto-optiques et, enfin, une lentille convergente pour focaliser le faisceau sur un photodétecteur. La partie analogique recouvre l'élaboration complète d'une voie électronique d'émission ainsi que celle d'une voie de réception.

Le milieu d'interaction a fait l'objet d'une sélection parmi les matériaux les plus courants avec le meilleur compromis Bande passante/Atténuation. Le composant retenu a été le Molybdate de Plomb $\left(\mathrm{PbMoO}_{4}\right)$ qui fonctionne très correctement sur l'octave de $150 \mathrm{MHz}$ à $300 \mathrm{MHz}$ et possède un coefficient d'atténuation acoustique $f$ de $1,5 \times 10^{-5} \mathrm{~dB} / \mathrm{cm}^{-\mathrm{MHz}^{2}}$. Une telle largeur de bande nous permet de prévoir l'utilisation de quatre canaux de traitement en parallèle possédant chacun, une bande de modulation d'environ $10 \mathrm{MHz}$. Le coefficient $f$ va déterminer la largeur utile maximale a du faisceau lumineux. Si l'atténuation sur toute la longueur d'interaction est limitée à $\mathrm{l} \mathrm{dB}$, la largeur a devra être réduite à environ $7,5 \mathrm{~mm}$ (dans le cas le plus défavorable, c'est-à-dire à $300 \mathrm{MHz}$ ). Cette dernière valeur ainsi que la bande passante $\delta f$ du canal considéré vont définir la longueur maximale du filtre transversal directement réalisable selon la relation [11]:

$$
\delta f<\left(N_{\text {cel }} \cdot v\right) /(2 \cdot a) \text { où } v \text { est la vitesse acoustique } .
$$

Ceci donne, pour un nombre $N_{\text {cel }}=16$ cellules (donc un filtre transversal de longueur maximale 16), $\delta f=4 \mathrm{MHz}$, soit une fréquence d'échantillonnage de $8 \mathrm{MHz}$. Dans la configuration monovoie, le générateur de la fréquence porteuse délivre un signal de fréquence double nécessaire à la démodulation du signal de sortie par détection synchrone. Nous conservons ainsi les informations de signe sur le résultat.

LA PARTIE NUMÉRIQUE. - La partie numérique comprend principalement le générateur d'horloges, les cartes système, étalonnage, mémoire et l'interfaçage avec le micro-ordinateur dont le rôle essentiel est de gérer la configuration, tant pour les signaux que pour le mode de fonctionnement.

Vu la complexité et le nombre des signaux à gérer, afin de simplifier la réalisation du système, notre choix s'est porté sur les circuits logiques programmables (PLDs), plutôt que sur des circuits classiques. Parmi tous ceux disponibles nous avons préféré les circuits GAL 20V8 et 16V8 de chez LATTICE, qui sont à la fois configurables et reprogrammables avec un temps de réponse compatible avec les caractéristiques du pilote à réaliser [12]. 
Les cartes système contrôlent les séquences d'émission et de réception. Bien qu'assurant des fonctions apparemment différentes, ces cartes sont identiques et seul un signal $\mathbf{S}$ détermine leur nature signal ou résultat. L'intérêt de cette méthode réside dans le fait que les signaux de commande et le cycle de fonctionnement sont rigoureusement les mêmes. Le retard nécessaire à la prise en compte des échantillons résultat par rapport à l'émission des signaux d'entrée s'effectue simplement par un décalage des signaux d'horloge pour chacune des cartes système. Le générateur d'horloge, qui réalise cette dernière fonction, travaille à partir d'impulsions de fréquence de répétition $40 \mathrm{MHz}$ et délivre les différents signaux d'horloge avec le retard adéquat (Fig. 7). les autres cartes concernent les fonctionnements annexes du système tels que étalonnage, génération de signaux, interfaçage, etc..

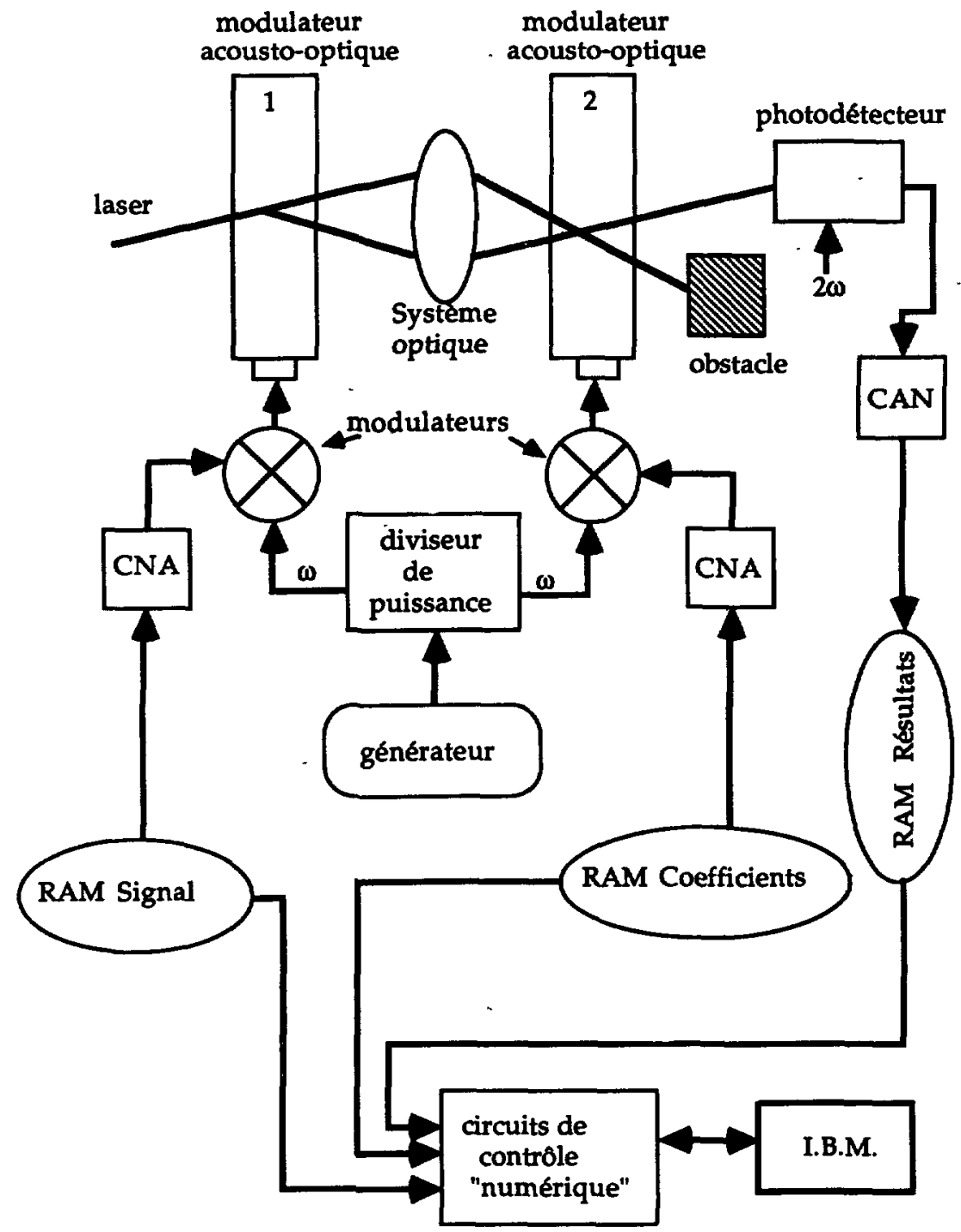

Fig. 7. - Schéma de principe de la manipulation.

[Experimental setup.] 
Différents modes de travail sont prévus aussi bien pour les calculs que pour le paramétrage du pilote. Les signaux de commande correspondants sont contrôlés par l'ordinateur via la carte d'interface au moyen d'un programme écrit en langage $C$. Les différentes options proposées sont le calcul, le générateur de signal qui permet entre autres de valider la convolution de base, et l'étalonnage afin d'effectuer la mise au point optique et de déterminer le retard et le gain de la chaîne de réception. Ces deux dernières fonctions sont réalisées à l'aide de la carte étalonnage.

\section{Résultats expérimentaux.}

Après avoir décrit le système, nous présentons quelques résultats obtenus pour les différentes fonctions décrites précédemment. Nous verrons, en premier lieu, la mise en cuvre des fonctions étalonnage et générateur de signal. Nous donnerons ensuite quelques résultats de calculs de filtrage FIR puis d'autres traitements réalisés avec les fonctions de base. Nous terminerons par l'analyse des performances du système.

CONVOLUTION DE BASE. - Cette opération, réalisée sous les fonctions étalonnage/générateur de signal, permet de vérifier le bon fonctionnement du système et d'effectuer la mise au point $\mathrm{du}$ banc optique et le réglage de l'électronique (gain, fréquence, retard). En mode étalonnage, la transformation concernée est l'auto-convolution d'une impulsion rectangulaire dont la largeur peut varier à volonté, suivant le réglage désiré. Les résultats exposés dans les figures 8 et 9 , obtenus en mode générateur de signal, correspondent à des signaux plus complexes (trains d'impulsions) et permettent d'apprécier la réponse globale du système. En particulier nous constatons que la durée du signal de sortie est identique à celle du signal d'entrée, mettant ainsi en évidence la compression d'un facteur deux dans le temps. Nous remarquons également une déformation de la base des triangles qui traduit un phénomène de traînage s'expliquant par la limitation globale de la bande passante du système.

EXEMPLES DE FILTRAGE TRANSVERSAL. - Nous présentons, maintenant des résultats de traitements par filtres FIR. Pour chacun d'eux, nous comparons les signaux obtenus par calcul et par simulation. Pour cette dernière, nous utilisons le logiciel DSPLAY de Burr-Brown.

Les courbes de la figure 10 correspondent à la simulation et l'expérimentation du filtrage (a) d'un signal carré de fréquence $500 \mathrm{kHz}$ (c) par un passe-bas, de fréquence de coupure $500 \mathrm{kHz}$, comportant 12 coefficients dont la réponse impulsionnelle est représentée en (b).

L'expérimentation d'autres types de filtres nécessite une longueur de filtre supérieure à 16 pour des conditions de filtrage efficaces. La réalisation de filtres de longueur supérieure nous a conduit à rechercher une méthode de décomposition de la réponse impulsionnelle en éléments plus simples. Nous avons pu ainsi effectuer le filtrage des harmoniques 3 et 5 d'un signal carré de fréquence $250 \mathrm{kHz}$ par un passe-bande à 39 coefficients (Fig. 11), soit un calcul en trois cycles $(3 \times 13)$.

CORRÉlATION ET PRODUIT MATRICIEL. - Si nous reprenons la convolution sous forme discrète et si nous inversons dans le temps l'un des deux signaux, en agissant sur le sens de lecture de la mémoire, la relation:

$$
s(i)=\sum_{j=0}^{N_{\mathrm{c}}-1} c(j) \cdot e(i-j)
$$

devient, si $N$ est le nombre total d'échantillons du signal inversé :

$$
s(N-\ell)=\sum_{j=0}^{N_{c}-1} c(j) \cdot e(\ell+j) .
$$



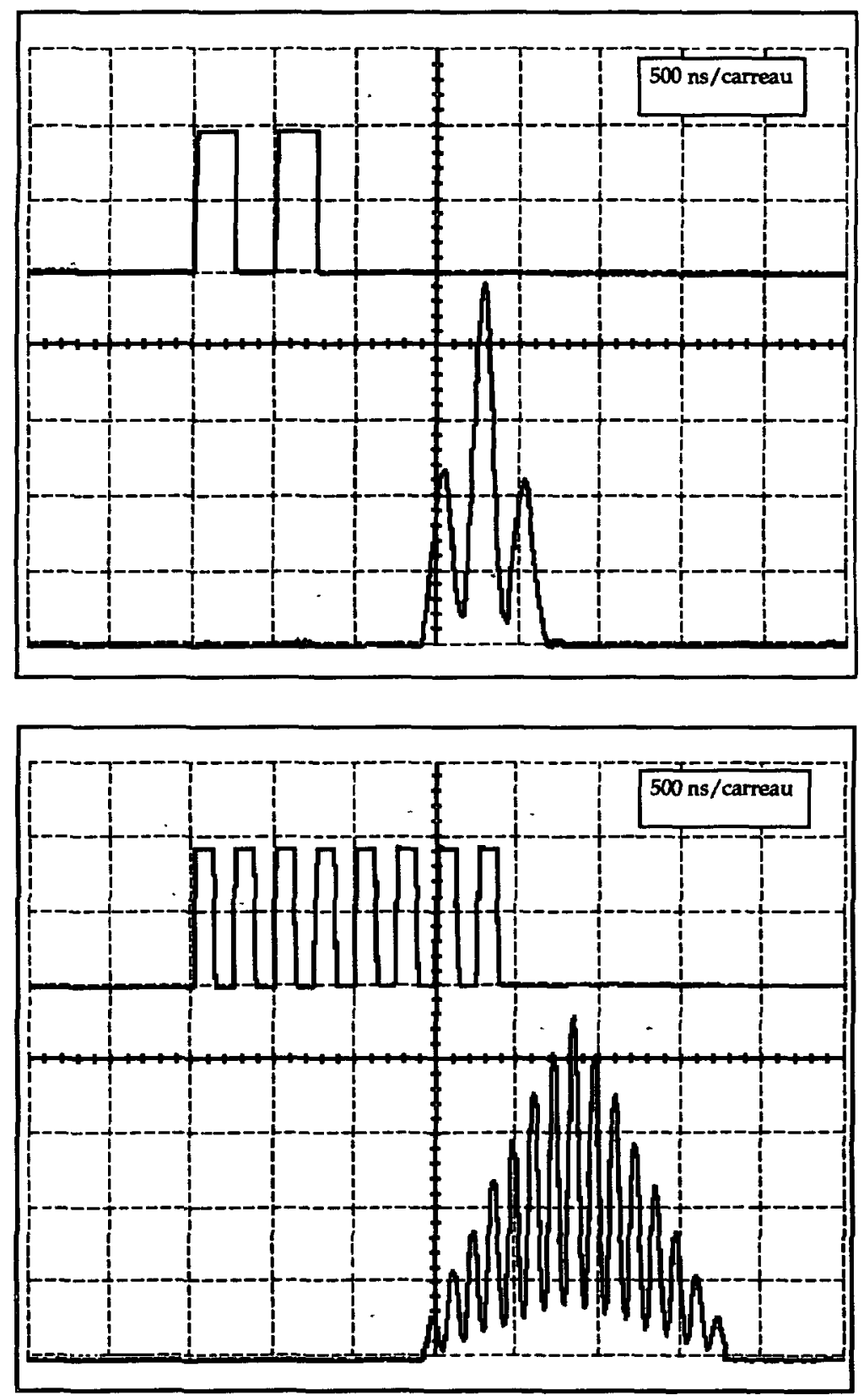

Figs. 8 et 9. - Autoconvolution de trains d'impulsions rectangulaires.

[Autoconvolution of rectangular pulses.]

Cette relation s'identifie au produit de corrélation des signaux $\{e\}$ et $\{c\}$, en remarquant que le sens de lecture des résultats devra, lui aussi, être inversé.

Pour illustrer cette transformation, considérons par exemple des signaux particuliers utilisés en détection au sens large, soient les codes complémentaires de Golay à huit moments [13]. Ils présentent la particularité de posséder une fonction d'auto-corrélation comportant un 

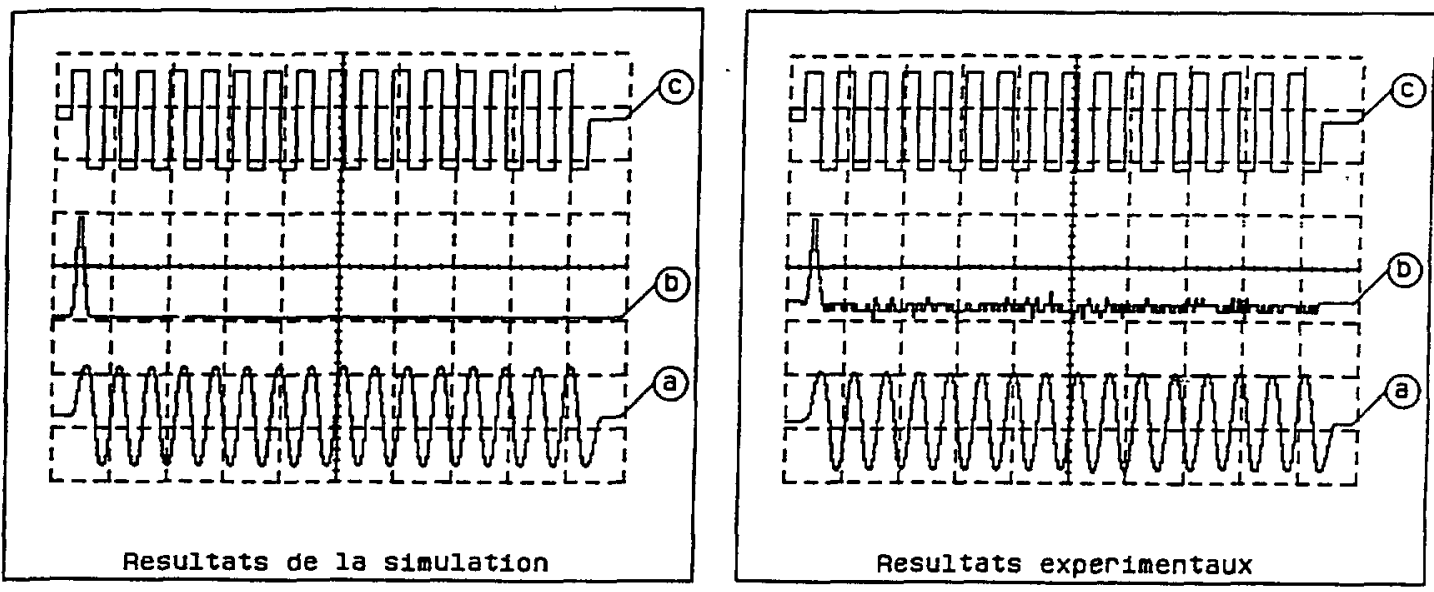

Fig. 10. - Filtre à 12 coefficients en 16 cellules. [12 coefficients filter with 16 cells.]
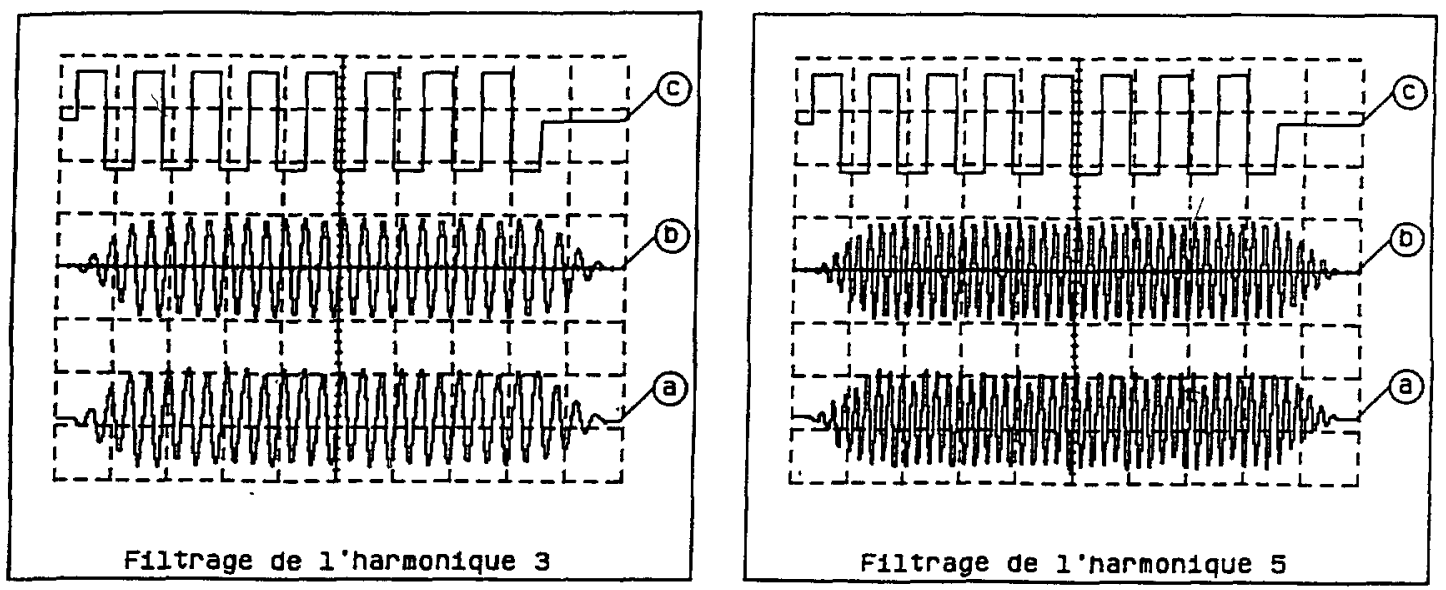

Fig. 11. - Filtrage des harmoniques d'un carré à $250 \mathrm{KHz}$.

[Filtering of harmonics of a $250 \mathrm{KHz}$ signal square wave.]

lobe central prononcé et des lobes secondaires non négligeables. Si l'on ajoute point par point les fonctions d'auto-corrélation correspondant à deux codes complémentaires les lobes secondaires disparaissent (Fig. 12). On observe toujours le facteur de compression de 2 pour le temps.

PRODUIT MATRICIEL. - Dans le domaine du traitement numérique par voie acousto-optique, le processeur matriciel est une des configurations les plus courantes.

Le produit de convolution sous forme discrète décrit complètement le produit d'une matrice par un vecteur lorsque celle-ci est une matrice de Toeplitz [14]. En effet, le produit 


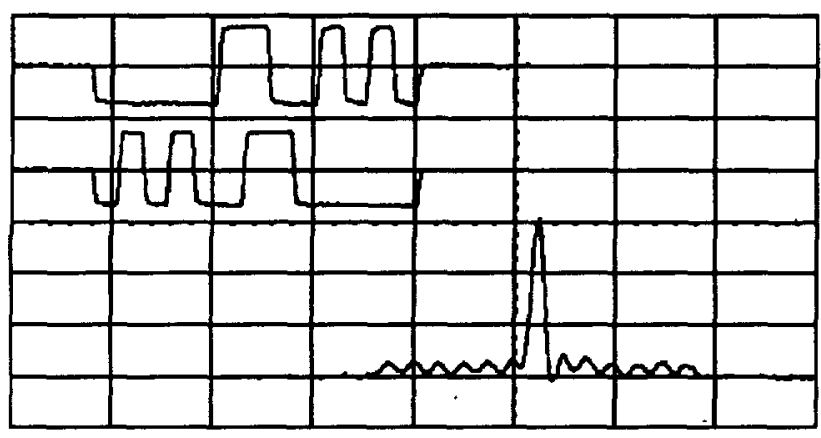

Code de Barker a 13 moments.

Autocorrélation de chaque code complémentaire de Golay
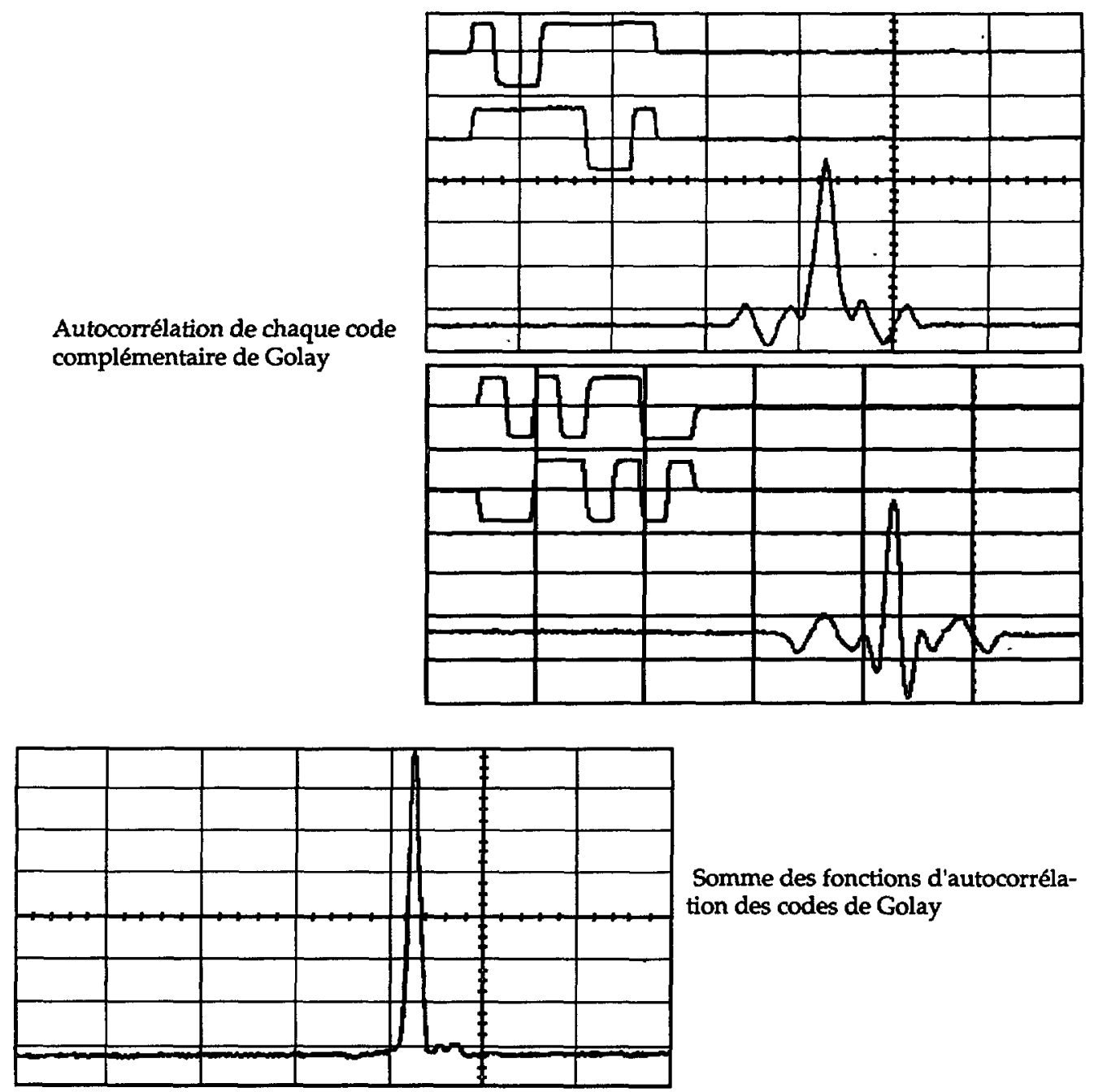

Somme des fonctions d'autocorrélation des codes de Golay

Fig. 12. - Fonction d'autocorrélation des codes de Barker et Golay.

[Autocorrelation of Barker and Golay codes.] 
d'une matrice $\left[a_{i j}\right]$ de dimension $5 \times 3$ par un vecteur $\left[b_{i}\right]$ à trois composantes correspond à la relation de convolution suivante :

$$
c(i)=\sum_{j} a(i, j) \cdot b(j) .
$$

\section{Evaluation du système.}

Nous allons ici caractériser globalement le système du point de vue dynamique, capacité et surtout vitesse de traitement. Cette analyse nous permettra ensuite de faire une comparaison avec la technologie la plus courante dans ce domaine, c'est-à-dire les circuits intégrés numériques.

DYNAMIQUE ET CAPACITÉ DE TRAITEMENT. - Le réglage du gain de la chaîne de sortie en mode étalonnage nous a permis de mesurer la dynamique analogique du convoluteur acoustooptique. En effet, l'auto-convolution d'une impulsion rectangulaire de largeur 16 périodes d'horloge correspond à la mesure de niveau maximal. La mesure du rapport signal à bruit, en supposant le système linéaire, est identique à celle de la dynamique analogique et nous donne une valeur voisine de $50 \mathrm{~dB}$. Ceci est donc compatible avec le choix de la définition des convertisseurs analogiques/numériques et numériques/analogiques qui est de 8 bits (soit 256 niveaux).

La capacité de traitement ne dépend principalement que de la taille des mémoires utilisées. Dans notre cas, nous avons prévu une capacité maximale de 2048 échantillons, ce nombre pouvant être configuré facilement à 1024,512 ou 256 octets. Le nombre maximal de coefficients directement utilisable est de 16. Cette limite est facilement contournable en décomposant les filtres de taille supérieure en éléments simples de longueur inférieure.

VITESSE DE CALCUL. - Le paramètre qui caractérise le mieux la rapidité du système est le temps moyen de calcul d'un point $T_{\mathrm{mp}}$. La sous-passe étant la composante de base de l'algorithme, c'est à partir de celle-ci que nous allons calculer $T_{\mathrm{mp}}$. En considérant la période d'horloge $T_{\mathrm{b}}$ du système, la durée d'une sous-passe et le cycle d'initialisation de celle-ci $\left(4 \times T_{b}\right)$, on obtient :

$$
T_{\mathrm{mp}}=\left(N_{\mathrm{cel}}+4\right) \cdot T_{\mathrm{b}} /\left(N_{\mathrm{cel}}-N_{\mathrm{c}}+1\right) .
$$

Notamment, pour une longueur de filtre $N_{\mathrm{c}}$ unitaire, $T_{\mathrm{mp}}$ vaut $156 \mathrm{~ns}$, pour $N_{\mathrm{c}}=8$, $T_{\mathrm{mp}}=278 \mathrm{~ns}$ et pour $N_{\mathrm{c}}=16, T_{\mathrm{mp}}=2,5 \mu \mathrm{s}$.

COMPARAISON AVEC LES CIRCUITS INTÉGRES. - Les valeurs calculées précédemment n'ont d'intérêt que dans la mesure où elles permettent d'effectuer une comparaison avec les circuits intégrés numériques du commerce. En effet, pour ces derniers, la grandeur habituellement annoncée est le temps de cycle $T_{\mathrm{c}}$ qui correspond à la durée d'un calcul élémentaire (multiplication-accumulation) pour un coefficient du filtre. Ainsi, pour un filtre de longueur $N_{\mathrm{c}}$, avec un cycle d'initialisation, le temps de calcul moyen d'un point $T_{\text {ic }}$ est de $\left(N_{\mathrm{c}}+1\right) \times T_{\mathrm{c}}$. Nous pouvons écrire, d'après le calcul de $T_{\mathrm{mp}}$ et de ce qui précède, que la condition pour que le système hybride présenté soit plus rapide se ramène à :

$$
T_{\mathrm{c}}>20 \cdot T_{\mathrm{b}} /\left[\left(17-N_{\mathrm{c}}\right) \cdot\left(N_{\mathrm{c}}+1\right)\right] .
$$

Le cas optimal pour lequel $N_{\mathrm{c}}=8$ donne pour la valeur limite $T_{\mathrm{c}}=31 \mathrm{~ns}$; le cas le plus défavorable, $N_{\mathrm{c}}=16$, donne $147 \mathrm{~ns}$.

Les autres points de comparaison sont la longueur maximale pour un filtre, la précision, et la souplesse d'utilisation. Les circuits classiques admettent un nombre de coefficients assez 
grand et sont éventuellement cascadables. Dans notre cas, nous avons vu que ce nombre peut, également, être facilement étendu à une valeur quelconque. Le point faible du montage réside dans la précision limitée à 8 bits si l'on veut conserver la vitesse de calcul. Cependant, dans beaucoup de cas, elle est suffisante et comparable à celle de certains appareils de mesure. Par contre, l'utilisation d'un ordinateur pour paramétrer le montage, permet par modification d'un programme écrit en langage évolué classique (le $C$ dans notre cas), une grande souplesse d'utilisation. Ceci se rapproche d'un système de développement intégré.

\section{Améliorations du système.}

La première amélioration qui a été réalisée est l'extension du nombre de coefficients des filtres par la mise en cascade de plusieurs cycles de calculs. Pour cela, on tire profit du surdimensionnement de la mémoire coefficient. Cela permet de stocker tous les coefficients qui décrivent le filtre et d'effectuer les différents cycles par commutation de plans mémoire.

Afin d'optimiser la rapidité de traitement, plusieurs points peuvent être améliorés. En premier lieu, la fréquence d'horloge peut être augmentée de $25 \%$ (horloge de base portée à $10 \mathrm{MHz}$ ) par l'utilisation de GAL plus rapides, maintenant disponibles. De même, l'emploi de réseaux logiques programmables à haute densité d'intégration (ASICs par exemple) permettrait, par une meilleure utilisation des circuits, de simplifier considérablement le système avec, comme conséquence, la réduction à 1 du nombre de cycles d'initialisation. En dernier lieu, le temps de calcul peut être encore divisé par 2. Si pour l'échantillonnage des résultats, au lieu d'une horloge de même fréquence que celle d'émission on utilise une fréquence double, alors nous pouvons effectuer le calcul complet en une passe unique. En effet, dans ces conditions, au résultat d'une parité succède le résultat de l'autre parité au cours de la même sous-passe.

Si l'on met en œuvre toutes les améliorations proposées, le nouveau temps moyen de calcul par point, $T_{\mathrm{mpn}}$, s'exprime par :

$$
T_{\mathrm{mpn}}=17 \cdot T_{\mathrm{b}}^{\prime} /\left(33-2 \cdot N_{\mathrm{c}}\right)
$$

et les nouvelles limites sur les temps de cycle des circuits intégrés du commerce deviennent :

$$
\begin{array}{lll}
- \text { pour } & N_{\mathrm{c}}=8 & T_{\mathrm{c}}>11 \mathrm{~ns} \\
- \text { pour } & N_{\mathrm{c}}=16 & T_{\mathrm{c}}>100 \mathrm{~ns} .
\end{array}
$$

\section{Conclusion.}

Les performances obtenues à l'issue de cette étude sont très prometteuses et nous permettent d'envisager une suite à ces travaux. En effet, il reste à exploiter les possibilités de fonctionnement multicanal du système acousto-optique qui autorise un traitement parallèle sur plusieurs fréquences et permet d'augmenter la puissance de calcul du système. Elles feront l'objet d'une extension du travail dans lequel seront également développées les applications. Une adaptation du pilote précédent permettra notamment la réalisation de filtrage à réponse impulsionnelle infinie (IIR) et de filtrage adaptatif (filtres de Wiener, Kalman) qui nécessitent une contre-réaction. 


\section{Bibliographie}

[1] Cafarella, « Programmable Transversal Filters : Applications and Capabilities », IEEE Ultrasonics Symposium (1987).

[2] Reticon E. G. \& G., «Analog Signal Processing Integrated Circuits », Catalog (1987).

[3] Dieulesaint, Royer, "Ondes élastiques dans les solides" (Masson, 1974).

[4] BESson, "Les composants », T.L.E. (août-septembre 1985).

[5] Goutin, Logette, Rouvaen, BridouX, « Design and Applications of an Hybrid Acousto-Optic Processor ", IEEE Ultrasonics Symposium (1990).

[6] Young, Sh7-Kay Yao, «Design Considerations for Acousto-Optic Devices », Proc. IEEE, 69, 1 (1981).

[7] KorPel, "Acousto-Optics - A Review of Fundamentals », Proc. IEEE, 69, 1 (1981).

[8] WAXIN, "Etude et réalisation de composants acoustiques de traitement de signal », Thèse de doctorat $3^{\mathrm{e}}$-cycle (1980).

[9] Goutin, Logette, Rouvaen, Bridoux, "Algorithm for Acousto-Optic Processing of Digital Signals », Ac. Lett. 14 (1991) 10.

[10]'Goutin, «Etude et réalisation d'un processeur acousto-optique hybride pour le traitement - numérique du signal », Thèse de doctorat en électronique (1990).

[11] Pfeiffer, Meyrueis, «Une nouvelle voie vers les hyper-ordinateurs phototoniques: les processeurs à modulation spatiale de lumière ", Spectra 14 (1986) 111.

[12] LATTICE SEMICONDUCTOR CORPORATION, “G.A.L. Databook », Spring 1988.

[13] LAM, "Microcomputer-Based Digital Pulse Compression System for Ultrasonic N.D.T.», Ultrasonics 25 (1987).

[14] CASAsent, "Acousto-optic Transducers in Iterative Optical Vector-Matrix Processors », Appl. Opt. 21 (1982) 10. 